International Journal of Biomedicine I June 2019 - Volume 9, Issue Suppl_1: Abstracts From the Second Russian International Conference "Cryo-electron microscopy 2019: achievements and prospects"

POSTER ABSTRACT PRESENTATIONS

SESSION TITLE: STRUCTURE AND FUNCTIONS OF THE TRANSCRIPTION AND TRANSLATION APPARATUS OF THE CELL

DOI: 10.21103/IJBM.9.Suppl_1.P17

\title{
Abstract P-17: Structural and Functional Insights into Eukaryotic Translation Reinitiation and Ribosome Recycling Orchestrated by eIF2D and MCT-1/DENR
}

\author{
Desislava S. Makeeva ${ }^{1}$, Elena A. Stolboushkina ${ }^{2}$, Ivan B. Lomakin ${ }^{3}$, Sergey E. Dmitriev ${ }^{1}$ \\ ${ }^{1}$ Belozersky Institute of Physico-Chemical Biology, Lomonosov Moscow State University, Moscow, \\ Russia; ${ }^{2}$ Institute of Protein Research, Russian Academy of Sciences, Pushchino, Russia; ${ }^{3}$ Department of \\ Molecular Biophysics and Biochemistry, Yale University, New Haven, USA
}

The last stage of translation cycle is ribosome recycling which occurs after completed polypeptide is released. This step comprises dissociation of $60 \mathrm{~S}$ ribosomal subunit, followed by tRNA and mRNA removal from the 40S-tRNA-mRNA post-termination complex. However, in some cases unrecycled 40S subunit is able to resume scanning on the same mRNA and initiate translation at the next start codon, thus resulting in translation reinitiation.

As previously shown in a reconstituted mammalian system, eukaryotic translation factor eIF2D and a heterodimer of two oncoproteins, MCT-1 and DENR, can operate with tRNA in the ribosomal Psite in two different modes, either stabilizing its binding to a non-canonical 48S initiation complex, or facilitating its removal from the post-termination 40S subunit followed by mRNA dissociation. However, the precise role of these proteins in living cells remained unclear.

Using in vitro translation systems prepared from S.cerevisiae mutant strains, we demonstrated that yeast orthologs of eIF2D, MCT-1 and DENR (TMA64, TMA20 and TMA22, respectively) prevent illegitimate reinitiation at the 3' untranslated regions of reporter mRNAs, and reduce reinitiation on mRNAs with uORF. Here we discuss how the recently solved X-ray and cryo-EM structures of the human MCT-1/DENR or eIF2D on the 40S ribosomal subunit provide mechanistic insights into this activity.

C-terminal regions of eIF2D and DENR contain SUI1 domain, which is also present in SUI1/eIF1, a factor controlling stringency of AUG recognition during translation initiation. Structural data indicate that, similarly to eIF1, the SUI1 domains of DENR and eIF2D are positioned in the P-site, modulating tRNA binding and codon-anticodon interaction. Other portions of the eIF2D or MCT-1/DENR molecules, including DUF1947 and PUA domains, provide an interface for tRNA CCA-end binding and occupy landing sites for canonical translation initiation factors eIF2 and eIF3 on the 40S subunit. 
Based on these data, we proposed a model of how eIF2D (TMA64) and MCT-1/DENR (TMA20/TMA22) control translation reinitiation by forcing 40S recycling and preventing binding of eIF1, eIF2 and eIF3 to the post-termination $48 \mathrm{~S}$ complex.

Key Words: protein synthesis $\bullet$ translation reinitiation and ribosome recycling $\bullet$ eIF2D/ligatin $\bullet$ MCTS1

Sources of Funding: This work was supported by the Russian Science Foundation (grant RSF 18-1400291 to S.E.D.) and the Russian Foundation for Basic Research (grant RFBR 18-04-01331 to E.A.S.)

International Journal of Biomedicine. 2019;9 Suppl 1: S24. doi: 10.21103/IJBM.9.Suppl_1.P17

(C)2019 International Medical Research and Development Corporation 